\title{
BMJ Open Protocol of a randomised controlled trial on the efficacy of medication optimisation in elderly inpatients: medication optimisation protocol efficacy for geriatric inpatients (MPEG) trial
}

Kenya le (D) , ,,2 Masanori Hirose, ${ }^{2}$ Tsubasa Sakai, ${ }^{1,2}$ Iori Motohashi, ${ }^{1,2}$ Mari Aihara, ${ }^{1,2}$ Takuya Otsuki, ${ }^{1,2}$ Ayako Tsuboya, ${ }^{3}$ Hiroshi Matsumoto, ${ }^{3}$ Hikari Hashi, ${ }^{3}$ Eisuke Inoue, ${ }^{4}$ Masaki Takahashi, ${ }^{5}$ Eiko Komiya, ${ }^{3}$ Yuka Itoh, ${ }^{3}$ Tomoya Tsuchida (1) ,2 Eri Kurosu, ${ }^{1,2}$ Steven M Albert, ${ }^{6}$ Chiaki Okuse, ${ }^{1,2}$ Takahide Matsuda ${ }^{2}$

To cite: le K, Hirose M, Sakai T, et al. Protocol of a randomised controlled trial on the efficacy of medication optimisation in elderly inpatients: medication optimisation protocol efficacy for geriatric inpatients (MPEG) trial. BMJ Open 2020;10:e041125. doi:10.1136/ bmjopen-2020-041125

- Prepublication history and additional material for this paper is available online. To view these files, please visit the journal online (http://dx.doi.org/10. 1136/bmjopen-2020-041125).

Received 31 May 2020 Revised 31 August 2020 Accepted 11 September 2020

Check for updates

(C) Author(s) (or their employer(s)) 2020. Re-use permitted under CC BY-NC. No commercial re-use. See rights and permissions. Published by BMJ.

For numbered affiliations see end of article.

Correspondence to

Dr Kenya le;

kenya.ie@marianna-u.ac.jp

\section{ABSTRACT}

Introduction Whether medication optimisation improves clinical outcomes in elderly individuals remains unclear. The current study aims to evaluate the effect of multidisciplinary team-based medication optimisation on survival, rehospitalisation and unscheduled hospital visits in elderly patients.

Methods and analysis We report the protocol of a single-centre, open-label, randomised controlled trial. The enrolled subjects will be medical inpatients, aged 65 years or older, admitted to a community hospital and receiving five or more regular medications. The participants will be randomly assigned to receive either an intervention for medication optimisation or the usual care. The intervention will consist of a multidisciplinary team-based medication review, followed by a medication optimisation proposal based on the Screening Tool of Older Persons' potentially inappropriate Prescriptions/Screening Tool to Alert doctors to the Right Treatment criteria and an implicit medication optimisation protocol. Medication optimisation summaries will be sent to primary care physicians and community pharmacists on discharge. The primary outcome will be a composite of death, unscheduled hospital visits and rehospitalisation until 48 weeks after randomisation. Secondary outcomes will include each of the primary endpoints, the number of prescribed medications, quality of life score, level of long-term care required, drugrelated adverse events, death during hospitalisation and falls. Participants will be followed up for 48 weeks with bimonthly telephone interviews to assess the primary and secondary outcomes. A log-rank test stratified by randomisation factors will be used to compare the incidence of the composite endpoint. The study was initiated in 2019 and a minimum of 500 patients will be enrolled.

Ethics and dissemination The study protocol has been approved by the Institutional Ethical Committee of St. Marianna University School of Medicine (No. 4129). The

\section{Strengths and limitations of this study}

The medication optimisation protocol efficacy for geriatric inpatients trial is a large randomised controlled trial that will examine the efficacy of multidisciplinary team-based medication optimisation on patient-oriented outcomes.

- The study will be adequately powered to examine the efficacy of medication optimisation protocol in elderly inpatients with a 48-week follow-up period.

- The multidisciplinary team-based intervention incorporates both explicit and implicit deprescribing criteria to enhance the efficacy of medication optimisation in elderly inpatients.

- The open-label design of this study has limitations; however, it will provide a rationale for future multicentre confirmatory studies.

results of the current study will be submitted to a peerreviewed journal.

Trial registration number UMIN000035265

\section{INTRODUCTION}

Polypharmacy is known to increase death rate, fall incidence and healthcare utilisation in elderly individuals. ${ }^{1-3}$ Potentially inappropriate medication lists (PIMs), aiming to reduce inappropriate medication prescriptions in elderly individuals, have been a mainstay of medication optimisation strategies. ${ }^{4}$ They can be used as an explicit criterion for medication reconciliation. Among the PIMs, the Screening Tool of Older Persons' potentially inappropriate Prescriptions/Screening Tool to Alert doctors to the Right Treatment 
(STOPP/START) is a widely accepted criterion, incorporating the list of medicines that are potentially harmful and those that should be prescribed for elderly individuals. ${ }^{5}$ A recent systematic review of randomised controlled trials of interventions to reduce polypharmacy using the STOPP criteria showed that the STOPP-based interventions are associated with reduced falls, emergency visits and medical costs, and short hospital stays. ${ }^{6}$ However, to date, no study has shown the effect of STOPP-based interventions on clinically important outcomes such as death and readmission rates. In reality, most adverse drug reactions are caused by drugs that are not included in such criteria. ${ }^{7}$ In addition, there could be both 'appropriate' and 'inappropriate' polypharmacy, depending on the patient background.

Recently, a more implicit criterion for polypharmacy, called the deprescribing protocol, has been expected to improve patient outcomes. Scott et al defined it as 'the systematic process of identifying and discontinuing drugs in instances in which existing or potential harms outweigh existing or potential benefits within the context of an individual patient's care goals, current level of functioning, life expectancy, values and preferences. ${ }^{, 8}$ The use of the deprescribing protocol has been indicated to reduce the number of prescription drugs, ${ }^{9}$ but whether the intervention improves significant patient-oriented outcomes, such as death, hospitalisation and falls, remains controversial. ${ }^{10-12}$ A Cochrane review pointed out that studies that failed to demonstrate the benefits of the deprescribing protocol had a follow-up period of less than 1 year, which may not be sufficient to identify the true effect of the deprescribing protocol. ${ }^{13}$ Thus, a lack of evidence regarding the effect of the deprescribing protocol on patient-oriented outcomes could be attributed to methodological limitations in previous studies.

\section{Objectives}

In this study, we aim to evaluate the effect of multidisciplinary team-based medication optimisation process, using both explicit and implicit criteria, on survival, rehospitalisation and unscheduled hospital visits in elderly inpatients.

\section{METHODS AND ANALYSIS \\ Medication optimisation protocol efficacy for geriatric inpatients trial design}

This is a single-centre, open-label, randomised controlled trial with a two-arm parallel design. Figure 1 depicts the flow diagram of the progress through various phases of the study. The current trial protocol follows the Standard Protocol Items: Recommendations for Interventional Trials guidelines, developed to provide a standardised guidance for recommended items to be included in a clinical trial protocol. ${ }^{14}$ The study was approved by the Institutional Ethics Committee of St. Marianna University School of Medicine (no. 4129).

\section{Study setting and eligibility criteria}

The present trial will be conducted in patients in the medical wards of a university-affiliated community hospital. Patients admitted to the medical wards will be screened for study eligibility by hospital receptionists, medical ward-based pharmacists, and the principal investigators or coinvestigators.

The eligibility criteria for participants are as follows:

1. Medical inpatients.

2. Aged 65 years or older.

3. Taking five or more regularly prescribed medications.

4. Predicted length of hospital stay after admission: 1 week or longer.

Exclusion criteria include the following: inability to take medications orally; life expectancy of less than 1 month based on attending physician's clinical judgement; and attending physicians disagreeing on study participation. In the current study, a regularly prescribed medication is defined as 'any form of prescribed oral medications recorded in the participant's medical record handbook, a referral letter, or electronic medical record over 28 days or longer at the time of hospital admission.' Drugs that are used 'as needed' will not be counted in regular medications.

\section{Interventions}

Participants will be randomly assigned to receive either a medication optimisation intervention or the usual care. Both groups will be subjected to medication reconciliation by ward-based pharmacists using data provided by the medical record handbook, patient/family or a referral letter, along with the usual care from their attending physicians. For those assigned to the intervention group, the multidisciplinary deprescribing team, which will consist of a physician and a pharmacist, will conduct the medication optimisation intervention within 48 hours of allocation. Ward-based nurses will be consulted by the deprescribing team, as required, to collect any information necessary for the medication optimisation proposal, including patient preference and medication adherence. All members of the deprescribing team will receive standardised instruction and guidance in advance. In addition, monthly deprescribing team meetings and casebased reflection for selected cases during the previous month will be held for monitoring and quality control of interventions.

Overall, the intervention will consist of a medication review, followed by the development of a medication optimisation proposal based on the STOPP/ START criteria ${ }^{5}$ and a medication optimisation protocol (figure 2). First, the study participant's baseline data (age, sex, medical history, comorbid conditions, height, weight, blood pressure, pulse rate, oxygen saturation, body temperature, estimated glomerular filtration rate (eGFR), serum sodium level, serum potassium level, and regularly prescribed medications) will be collected via a chart review and entered into a computer-based medication optimisation support system, developed specifically 


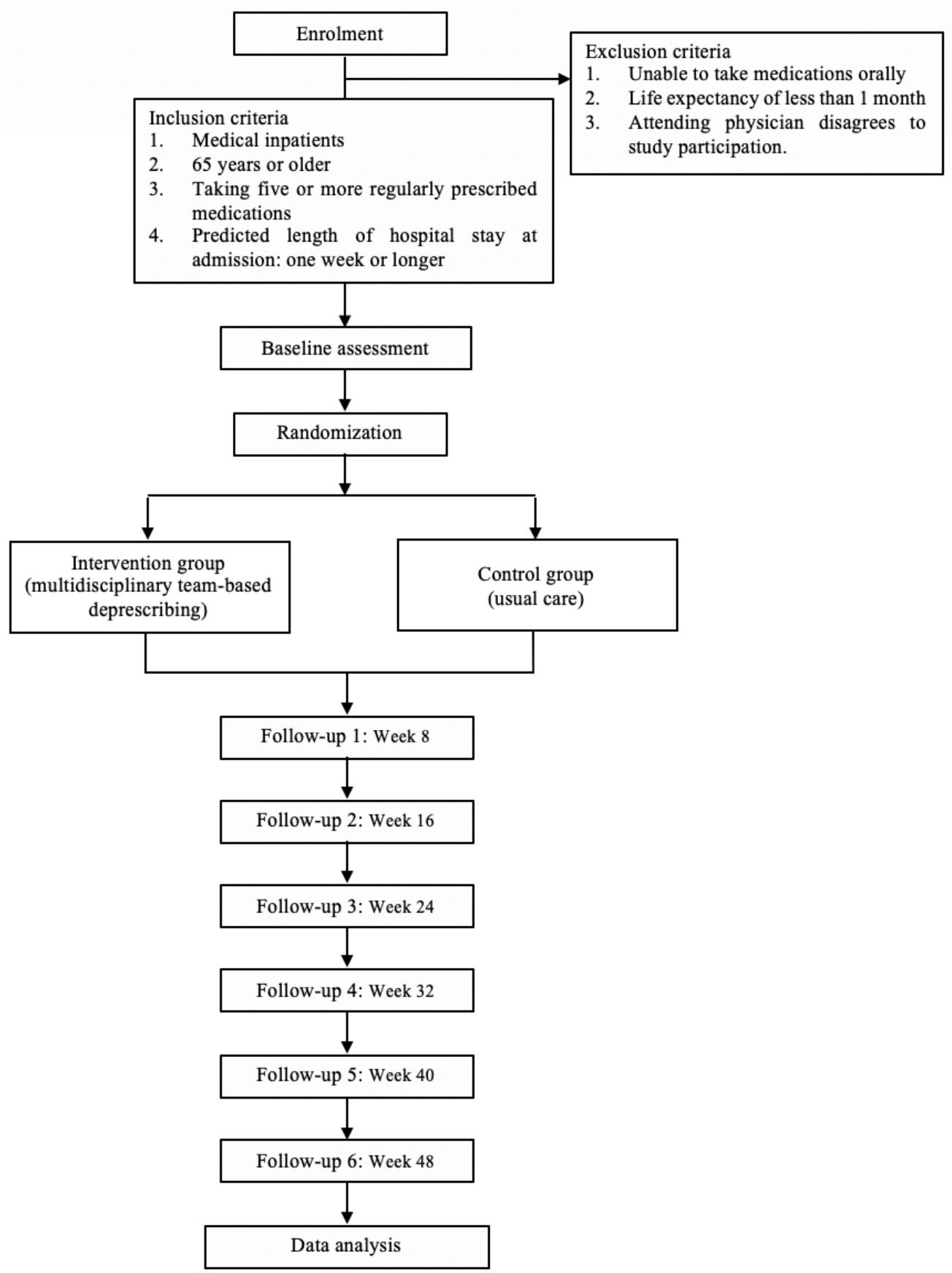

Figure 1 Flow chart summarising the MPEG trial procedure. MPEG, medication optimisation protocol efficacy for geriatric inpatients.

for the trial. The medication optimisation support system will automatically generate a draft proposal according to the STOPP/START criteria. ${ }^{5}$ After reviewing the draft proposal, the deprescribing team will conduct a step-bystep discussion based on the medication optimisation protocol (figure 3), involving the following steps per the algorithm proposed by Scott $e t a l^{8}$

1 . Does the prescription have an appropriate indication? All efforts will be made to ensure the indications for each drug. If no clear indication is confirmed or the drug is prescribed as a result of a prescribing cascade (eg, proton pump inhibitor to reduce gastrointestinal adverse effects associated with non-steroidal antiinflammatory drugs), the deprescribing team will discuss whether the drug should be deprescribed.

2. Does the harm outweigh the potential benefits? Study participants' symptoms and laboratory results will be reviewed to determine any adverse effect that outweighs the expected benefits of the prescribed drug (eg, calcium channel blocker in a patient with orthostatic hypotension and recurrent falls).

3 . For a symptomatic medication, does the patient currently have the target symptom? 


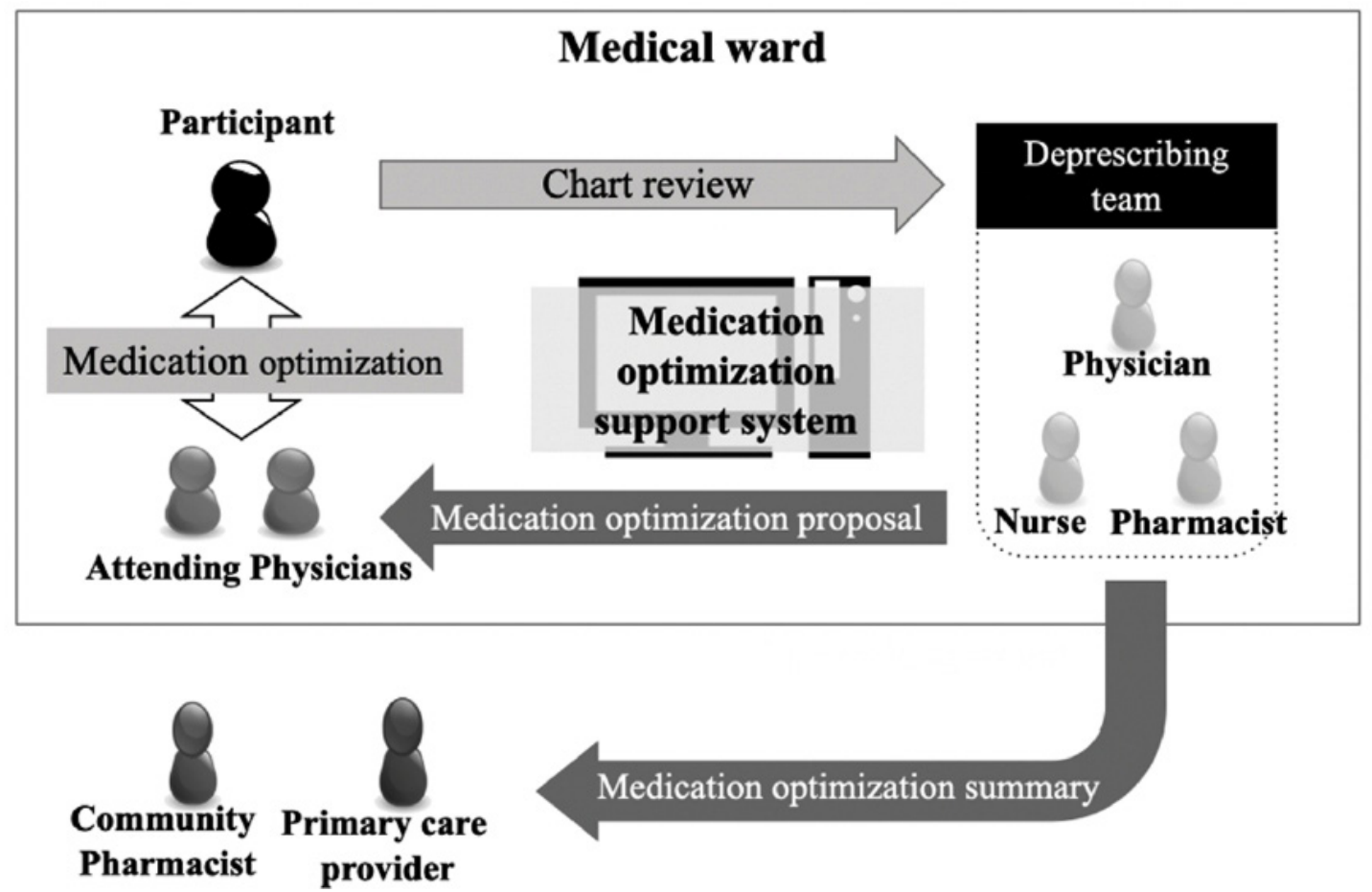

Figure 2 Scheme of multidisciplinary team-based medication optimisation intervention.

Symptomatic medications that control active symptoms to maintain quality of life (eg, painkillers and antiemetics) will be evaluated for their necessity. If the symptom is mild or intermittent or the drug is deemed ineffective, cessation, dose reduction or 'as-needed' use of the corresponding drug will be discussed.

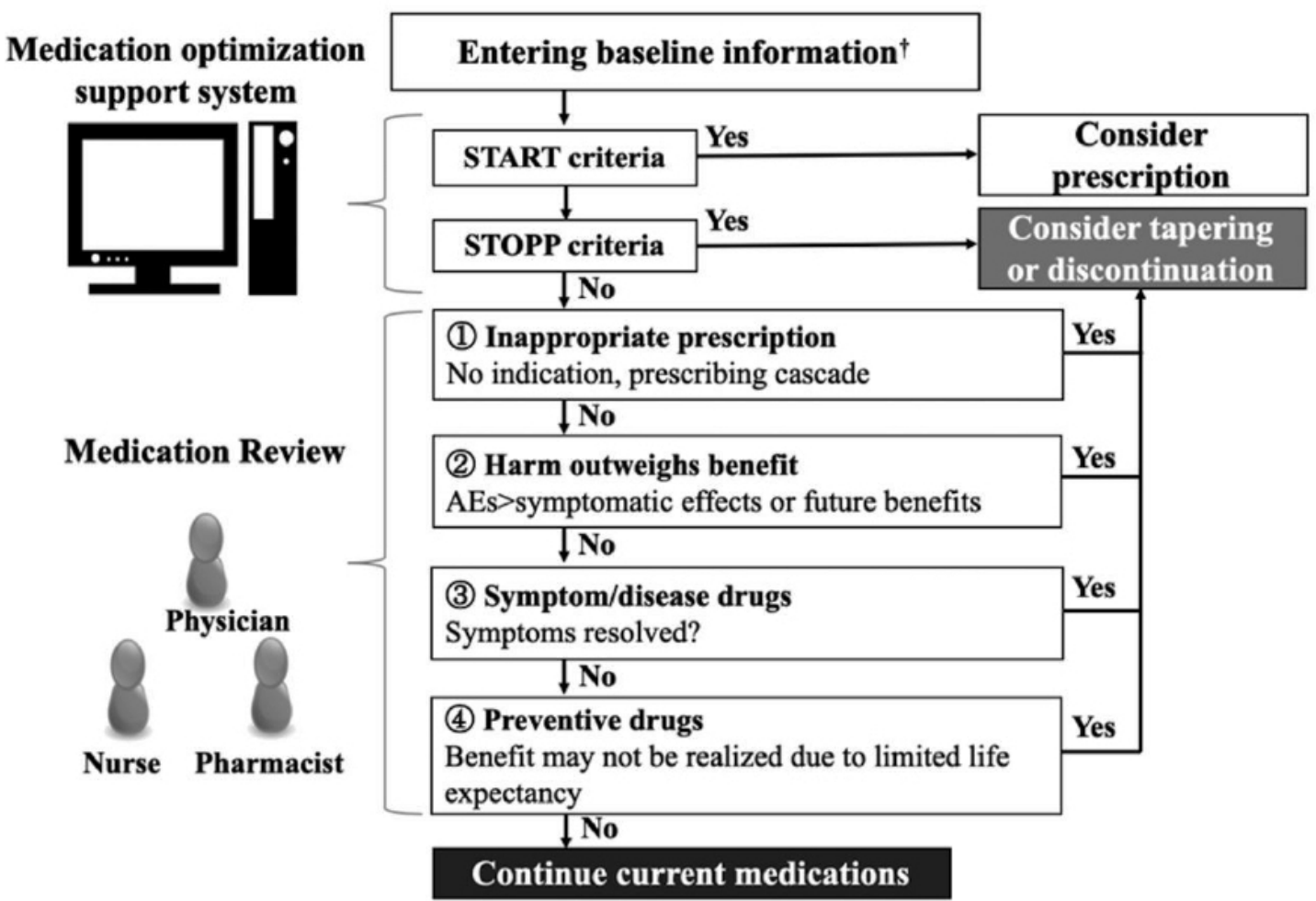

Figure 3 Medication optimisation protocol for the MPEG trial. †Baseline information includes study participants' age, sex, medical history, comorbid conditions, height, weight, blood pressure, pulse rate, oxygen saturation, body temperature, estimetaed glomerular filtration rate (eGFR), serum sodium level, serum potassium level and regularly prescribed medications. STOPP/START, Screening Tool of Older Persons' potentially inappropriate Prescriptions/Screening Tool to Alert doctors to the Right Treatment . 
4. For a preventive medication, does the patient have enough life expectancy to expect benefit of preventive care?

Medications aimed to prevent the occurrence of disease (eg, statins and glucose-lowering drugs) will be considered for their benefits, the length of time required for the expected benefit, and the participant's preference and estimated life expectancy.

The rationale for the medication optimisation proposal will be explained and discussed in detail with each participant or next of kin (NOK). On the participant's agreement, the team will recommend the medication optimisation plan, including its rationale, to the participant's attending physician. Whether the proposal would be accepted or not will be left to the discretion of the participant and his/her attending physician, as a part of clinical judgement. The details of each medication optimisation proposal and the list of medications at discharge will be recorded to track adherence to the proposal.

Medication optimisation summary, including the reason for prescription modification and relevant precautions, will be sent to the study participant's primary care physician and community pharmacists on discharge.

\section{Outcomes}

Primary outcome

The primary outcome is a composite of all-cause death, unscheduled hospital visits and rehospitalisation until 48 weeks after randomisation. Time to the first occurrence of primary composite outcome will be recorded for the survival analysis. An unscheduled hospital visit is defined as an unexpected visit to the emergency department or outpatient clinic during the follow-up period owing to new or worsening symptoms, signs and concerns. Any rehospitalisation due to new or worsening symptoms, signs and concerns after first hospital discharge will be recorded. A hospital transfer will be deemed as continuation of hospitalisation rather than rehospitalisation.

\section{Secondary outcomes}

The following endpoints at the baseline, 24 weeks and 48 weeks postrandomisation will be assessed as secondary outcomes.

1. Number of regular and PIMs.

The number of prescribed medications listed in the participant's medical record handbook, referral letter or electronic medical record over a duration of 28 days or longer at the baseline, 24 weeks and 48 weeks postrandomisation will be considered as 'regular medication.' Any prescribed regular medication listed in the STOPP criteria $^{5}$ will be indicated as PIM, whose number at the baseline, 24 weeks and 48 weeks postrandomisation will be recorded simultaneously.

2. Level of long-term care required (LTC)

The level of LTC required, under the JapaneseLTC insurance system, will be assessed at the baseline, 24 weeks and 48 weeks postrandomisation. The levels will be assigned by the local government as follows: independent, support required 1 or 2 , and care required 1-5-where care level 5 implies the highest level of requirement for LTC and independent implies the lowest level of requirement. ${ }^{15}$

3. Health-related quality of life

Self-reported general health status will be recorded at three time points using EuroQol 5 dimensions 3-levels (EQ5D-3L). ${ }^{16}$ We will use the Japanese version of EQ5D-3L and a Japanese scoring system that have been found to be valid and reliable. ${ }^{17}$

In addition to the above-listed outcomes, the ones listed below, occurring within 48 weeks after randomisation, will be assessed including the event dates.

- All-cause death.

- All-cause death during initial hospitalisation.

- Unscheduled hospital visits.

- Re-hospitalisation.

- Drug adverse events.

Any potential drug-related adverse events will be determined by consensus among the deprescribing team and attending physicians and recorded according to the Japanese version of CTCAE V.4.0. ${ }^{18}$ Drug names, symptom-onset timing, severity, treatment, consequence and relevance to the intervention will be entered in the report form.

- Falls

For the current study, a fall was defined per Gibson et al: "unintentionally coming to the ground or some lower level and other than as a consequence of sustaining a violent blow, loss of consciousness, sudden onset of paralysis as in stroke or an epileptic seizure. ${ }^{19}$

\section{Sample size}

Ravn-Nielsen et al, who examined the effect of multifaceted pharmacist intervention in medical wards, demonstrated a $23 \%$ reduction in hazard risk in the composite outcome of readmission or ED visits within 180 days after inclusion, compared with that in usual care. ${ }^{20}$ They did not find a significant difference in mortality across the groups, although a 6-month follow-up period may not be sufficient to detect a true effect. Another study conducted at residential aged care facilities revealed the deprescribing group, compared with the usual-care group, demonstrated a 40\% mortality reduction within 12 months of randomisation. ${ }^{9}$ Based on these two trials and other related studies, ${ }^{21-24}$ in this study, the investigators agreed on the requirement of at least 500 cases to provide a power of $80 \%$, with a significance level at alpha $=0.05$, on the assumption of primary composite endpoint rates of $30 \%$ and $40 \%$ in the intervention and control groups, respectively, and a true HR of 0.75 while allowing for a $15 \%$ drop-out.

\section{Recruitment}

We will recruit 500 subjects in the medication optimisation protocol efficacy for geriatric inpatients (MPEG) trial, based on the above-mentioned sample size calculation, to 
detect a significant difference in the primary outcome. Participants will be recruited from six medical wards in the study site (Kawasaki Municipal Tama Hospital). Community pharmacy and regional primary care provider outreach and advertising were conducted by the principal investigator before the study. Advertisements included information on inclusion criteria and time commitment, description of the intervention, and the participants' chance of receiving intervention. Recruitment of participants in this trial was initiated in May 2019 and will last for 2 years or until target enrolment is reached. Multiple strategies have been adopted in the recruitment process. Local physicians and other healthcare providers, including nurses and ward-based pharmacists in the study site, have been requested to refer potential participants. Participants will be given a gift card as a reward for study participation.

\section{Allocation}

The study participants who meet the eligibility criteria will be allocated to the intervention and usual-care groups on a one-on-one basis. Randomisation will be conducted on request from the staff member responsible for recruitment, using the computer-generated allocation sequence as a part of HOPE eACReSS, a clinical data management system. The HOPE eACReSS, developed by Fujitsu, Tokyo, Japan, ensures allocation concealment, and the randomisation method uses stratified block randomisation under age group blocks of 65-74, 75-84 and 85 years and above. The randomisation result will be stored, printed and immediately reported to the staff member responsible for intervention on that day.

\section{Blinding}

The research assistant who performs the bimonthly telephone interview assessments will be blind to group allocation. The investigators, ward-based pharmacists, participants' attending physicians, participants and/or their NOK will be aware of group allocation.

\section{Data collection and management}

The detailed participant timeline, including schedule of enrolment, interventions and outcome measurements, is presented in table 1 . Demographic characteristics at

Table 1 Timetable of the MPEG trial

\begin{tabular}{|c|c|c|c|c|c|c|c|c|}
\hline & $\begin{array}{l}\text { Baseline } \\
\text { assessment }\end{array}$ & Enrolment & Follow-up 1 & Follow-up 2 & Follow-up 3 & Follow-up 4 & Follow-up 5 & Follow-up 6 \\
\hline Time point (week) & 0 & 0 & $8 \pm 2$ & $16 \pm 2$ & $24 \pm 2$ & $32 \pm 2$ & $40 \pm 2$ & $48 \pm 2$ \\
\hline Informed consent & $x$ & & & & & & & \\
\hline $\begin{array}{l}\text { Sociodemographic } \\
\text { characteristics }\end{array}$ & $x$ & & & & & & & \\
\hline Intervention & & $x$ & & & & & & \\
\hline \multicolumn{9}{|l|}{ Assessments } \\
\hline Subjective symptoms & $x$ & $x$ & $x$ & $x$ & $x$ & $x$ & $x$ & $x$ \\
\hline Adverse events & & & $x$ & $x$ & $x$ & $x$ & $x$ & $x$ \\
\hline Vital signs & $x$ & & & & & & & \\
\hline Falls & & & $x$ & $x$ & $x$ & $x$ & $x$ & $x$ \\
\hline $\begin{array}{l}\text { Laboratory findings } \\
\text { (eGFR, serum sodium } \\
\text { level, and serum } \\
\text { potassium level) }\end{array}$ & $x$ & & & & & & & \\
\hline $\begin{array}{l}\text { No of prescribed } \\
\text { medications }\end{array}$ & $x$ & & & & $x$ & & & $x$ \\
\hline $\begin{array}{l}\text { No of prescribed } \\
\text { potentially } \\
\text { inappropriate } \\
\text { medications }\end{array}$ & $x$ & & & & $x$ & & & $x$ \\
\hline
\end{tabular}

${ }^{*}$ The number of prescribed potentially inappropriate medications listed in the STOPP/START criteria. ${ }^{5}$ eGFR, estimated glomerular filtration rate; EQ5D-3L, EuroQoL 5-Dimensions 3-Levels; STOPP/START, Screening Tool of Older Persons' potentially inappropriate Prescriptions/Screening Tool to Alert doctors to the Right Treatment. 
enrolment, including age, sex, date of admission, race, medical history, comorbid conditions, smoking status, physical measurements on admission (height, weight and vital signs), level of LTC required and history of falls within 3 months, will be collected via a chart review and interview by a trained research nurse and the deprescribing team. Participants' baseline medication list and laboratory findings (eGFR, serum sodium level and serum potassium level) will also be assessed by reviewing the participants' medical record handbook, referral letter and electronic medical record. Participants will be followed up by a telephone interview every 8 weeks, through 48 weeks, to assess any incidence of primary and secondary outcomes. The follow-up telephone interview will be performed by a trained research assistant blinded to group allocation. If the study candidate is unable to respond to the telephone interview due to lack of capacity, a predetermined NOK will be contacted. The medication lists at 24 and 48 weeks will be sent from the relevant community pharmacist on request from the investigators.

\section{Statistical analysis}

The primary and secondary outcomes will be adjudicated using the intention-to-treat analysis. All randomised participants will be analysed. For those who discontinue the trial before completion, all efforts will be made to follow their primary and secondary endpoints over the study duration through phone calls and health record review, if permitted. Participants who do not experience any endpoint will be censored either when lost to follow-up or at the completion of follow-up.

For the primary endpoint, survival functions for each group will be estimated using the Kaplan-Meier method, and a log-rank test (two sided), stratified by age group, will be conducted for the primary comparison. Significance level will be set at 0.05 . In addition, a Cox proportional hazards model will be used to estimate the HR across groups. The secondary outcomes, each of all-cause death, unscheduled hospital visits and rehospitalisation until 48 weeks after randomisation, will be compared by stratified log-rank test, and the HR will be estimated using Cox proportional hazards models. Prespecified subgroup analyses of the primary endpoint of indicator diseases (heart failure, pneumonia, diabetes mellitus, ischaemic stroke and urinary tract infection) and indicator drug classes (antiplatelets, antihypertensives, antidiabetics and sedatives) will be conducted for the exploratory analyses. All statistical analyses will be performed using STATA/SE V.15.0 (StataCorp).

\section{Data monitoring}

Central monitoring will be conducted at least once a year to check protocol compliance. The monitoring report will be submitted to the president (chairman of the ethics committee) and the investigators. Audit will be conducted, if the principal investigator deems it necessary, based on the monitoring report. There is no predetermined interim analysis for the current study. All study results will be analysed by a statistician in a deidentified form.

\section{Harms}

For the current trial, medication modification in the intervention group will be at the discretion of the participant and his/her attending physician, as a part of clinical practice. Thus, the risk of adverse events related to the intervention is not expected to significantly deviate from the usual care. However, any adverse event during the study period will be recorded according to the Japanese version of patient-reported outcome common terminology criteria for adverse events (PRO-CTCAE). ${ }^{18}$ Investigators will report to the president (chairman of the ethics committee) and the institutional data and safety monitoring board (I-DSMB) on all serious adverse events during the study period.

Serious adverse events in the MPEG trial are defined as follows:

1. All-cause death.

2. All-cause rehospitalisation.

Disability.

\section{Patient and public involvement}

The current trial will be conducted without direct patient involvement. The Institutional Ethics Committee of St. Marianna University School of Medicine includes patient representatives, charged with the responsibility to protect patient rights; thus, the MPEG trial protocol was reviewed by a patient representative. Besides the above review process, patients will not be invited to comment on the study design and interpretation of the study results. Patients were not involved in the writing of this manuscript.

\section{ETHICS AND DISSEMINATION}

\section{Ethics approval and consent to participate}

The current study protocol was approved by the Institutional Ethical Committee of St. Marianna University School of Medicine (No. 4129). Before study participation, oral and written explanations will be provided to all study candidates, and then written consent will be obtained (online supplemental file: patient consent form). If a study candidate is unable to provide consent due to a lack of capacity (ie, the researcher deems it inappropriate to obtain informed consent from the study candidate), the same will be obtained from the candidate's NOK. For this trial, a candidate's NOK is defined as the candidate's closest blood relative or one who is eligible to provide consent on behalf of the subject based on their mutual relationship. If the NOK cannot visit the hospital on that day, he/she will be contacted via telephone; oral consent is acceptable for study enrolment, provided that written consent can be obtained at a later date. If there is any revision to the study protocol that could affect the participants' decision to participate, the principal investigator 
will inform the participants and confirm their intent to continue their participation.

\section{Confidentiality}

The data obtained will be managed by the personal information manager, in accordance with the Act on the Protection of Personal Information, until 5 years after publication of the study results. Personal information, such as participants' name, date of birth, and hospital ID will be stored in a secure database (HOPE eACReSS) with password protection. All hard copies of data will be maintained in a locked cabinet. Data included in the HOPE eACReSS will be completely deidentified at the time of data entry to the web-based clinical research data management system.

\section{Dissemination plan and availability of data}

The results of the current study will be disseminated to healthcare providers, policy-makers and patients via presentations at local and national meetings, as well as by publication in a peer-reviewed journal. The datasets used and analysed during the current study are available from the corresponding author on reasonable request.

\section{DISCUSSION}

Herein, we describe the detailed methodology of the MPEG trial, a single-centre, open-label, randomised controlled trial with a two-arm parallel design. The main goal of this study is to demonstrate the efficacy and feasibility of multidisciplinary team-based intervention using both explicit and implicit criteria for medication optimisation. While the benefits of deprescribing have been increasingly highlighted and appear promising in terms of reducing inappropriate prescription, controversy regarding whether the deprescribing approach actually improves patient outcomes remains unresolved. ${ }^{10-12}$ In particular, there is a lack of evidence regarding the comparative effect of medication optimisation on clinically important outcomes such as survival, hospital admission and emergency department visits. Thus, the MPEG trial will primarily examine the effects of medication optimisation on these clinically important outcomes, as well as the number of prescribed medications, quality of life score, level of LTC required, drug-related adverse events, death during hospitalisation and falls.

Furthermore, the definition of deprescribing varies across studies, ranging from the use of explicit criteria, such as the STOPP/START criteria ${ }^{5}$ and Beers criteria, ${ }^{25}$ to the more implicit 'deprescribing protocol' approach proposed by Scott et al. ${ }^{8}$ Considering the strengths and weaknesses of explicit and implicit criteria, the complimentary use of both in the current study is expected to enhance the efficacy of the medication optimisation process in elderly individuals. Other methodological strengths of the MPEG trial include a relatively longer follow-up period and sufficient power to detect the true effect of intervention on clinically important outcomes.
Previous deprescribing trials that failed to reveal the effects of deprescribing were suggested to be limited by a shorter follow-up and a lack of sufficient power to detect the true effect. ${ }^{10-13}$ In this study, we will collect longitudinal data of approximately 500 patients for up to 48 weeks, allowing sufficient power and follow-up period to detect clinically important effects of the medication optimisation intervention.

Potential limitations of the current trial include the single-centre design and the potential of contamination due to its open-label nature; however, the results of this study will provide critical evidence regarding the effect of medication optimisation that may enhance the safety and efficient care of elderly multimorbid medical inpatients. In addition, the MPEG trial results will provide a rationale for future multicentre confirmatory studies.

\section{Author affiliations}

${ }^{1}$ Division of General Internal Medicine, Department of Internal Medicine, Kawasaki Municipal Tama Hospital, Kawasaki-shi, Kanagawa, Japan

${ }^{2}$ Division of General Internal Medicine, Department of Internal Medicine, St Marianna University School of Medicine, Kawasaki-shi, Kanagawa, Japan ${ }^{3}$ Department of Pharmacy, Kawasaki Municipal Tama Hospital, Kawasaki-shi, Kanagawa, Japan

${ }^{4}$ Showa University Research Administration Center, Showa University, Shinagawaku, Tokyo, Japan

${ }^{5}$ Division of Medical Informatics, St. Marianna University School of Medicine, Kawasaki-shi, Kanagawa, Japan

${ }^{6}$ Department of Behavioral and Community Health Sciences, University of Pittsburgh Graduate School of Public Health, Pittsburgh, Pennsylvania, USA

\section{Twitter Steven M Albert @SteveAlbert5}

Acknowledgements We acknowledge the work of research assistants Ruriko Kashikuma and Rei Miyazawa in participant recruitment and enrolment. We thank Junko Mita, Miyuki Kondo, and Satomi Tsuchiya for their assistance with data collection and data entry. We would also like to thank all study participants, the clinical pharmacists, St. Marianna University School of Medicine Clinical Research Data Centre, and nurses Shota Asamizu, Takahide Sadakata, and Chie Sekigawa for supporting this research.

Contributors Each author has contributed significantly to the study. Kl, MH, AT, HM, El, EK, YI and TM participated integrally in the study design. KI, El and MT contributed primarily to statistical analyses. All authors contributed to study protocol implementation, data acquisition and study data interpretation. KI and SMA drafted the initial manuscript, and all other authors, including $\mathrm{CO}$ and TM, read and approved the final manuscript.

Funding This work was supported by the Ministry of Education, Science, Sports and Culture, Grant-in-Aid for Young Scientists, 2018-2021 (grant number 18K15434, Kenya le).

\section{Competing interests None declared.}

Patient and public involvement Patients and/or the public were not involved in the design, or conduct, or reporting, or dissemination plans of this research.

Patient consent for publication Not required.

Provenance and peer review Not commissioned; externally peer reviewed.

Open access This is an open access article distributed in accordance with the Creative Commons Attribution Non Commercial (CC BY-NC 4.0) license, which permits others to distribute, remix, adapt, build upon this work noncommercially, and license their derivative works on different terms, provided the original work is properly cited, appropriate credit is given, any changes made indicated, and the use is non-commercial. See: http://creativecommons.org/ licenses/by-nc/4.0/.

\section{ORCID iDs}

Kenya le http://orcid.org/0000-0002-1387-0588

Tomoya Tsuchida http://orcid.org/0000-0002-8517-3941 


\section{REFERENCES}

1 Centers for Medicare \& Medicaid Services. Nhe fact sheet, 2015. Available: http://web.archive.org/web/20160325101526/https://www. cms.gov/research-statistics-data-and-systems/statistics-trends-andreports/nationalhealthexpenddata/nhe-fact-sheet.html [Accessed 30 May 2020].

2 Veehof L, Stewart R, Haaijer-Ruskamp F, et al. The development of polypharmacy. A longitudinal study. Fam Pract 2000;17:261-7.

3 Peterson L, Bazemore AW, Phillips RL, et al. Family physicians' present and future role in caring for older patients. Am Fam Physician 2009;80:1072.

4 By the American Geriatrics Society 2015 Beers Criteria Update Expert Panel. American geriatrics Society 2015 updated beers criteria for potentially inappropriate medication use in older adults. $J$ Am Geriatr Soc 2015;63:2227-46.

5 O'Mahony D, O'Sullivan D, Byrne S, et al. STOPP/START criteria for potentially inappropriate prescribing in older people: version 2. Age Ageing 2015;44:213-8.

6 Hill-Taylor B, Walsh KA, Stewart S, et al. Effectiveness of the STOPP/ START (screening tool of older persons' potentially inappropriate Prescriptions/Screening tool to alert doctors to the right treatment) criteria: systematic review and meta-analysis of randomized controlled studies. J Clin Pharm Ther 2016;41:158-69.

7 Verdoorn S, Kwint H-F, Faber A, et al. Majority of drugrelated problems identified during medication review are not associated with STOPP/START criteria. Eur J Clin Pharmacol 2015;71:1255-62.

8 Scott IA, Hilmer SN, Reeve E, et al. Reducing inappropriate polypharmacy: the process of deprescribing. JAMA Intern Med 2015;175:827-34.

9 Potter K, Flicker L, Page A, et al. Deprescribing in frail older people: a randomised controlled trial. PLoS One 2016;11:e0149984.

10 Christensen M, Lundh A. Medication review in hospitalised patients to reduce morbidity and mortality. Cochrane Database Syst Rev 2016;2:CD008986.

11 Johansson T, Abuzahra ME, Keller S, et al. Impact of strategies to reduce polypharmacy on clinically relevant endpoints: a systematic review and meta-analysis. Br J Clin Pharmacol 2016;82:532-48.

12 Cooper JA, Cadogan CA, Patterson SM, et al. Interventions to improve the appropriate use of polypharmacy in older people: a Cochrane systematic review. BMJ Open 2015;5:e009235.
13 Rankin A, Cadogan CA, Patterson SM, et al. Interventions to improve the appropriate use of polypharmacy for older people. Cochrane Database Syst Rev 2018;9:CD008165.

14 Chan A-W, Tetzlaff JM, Altman DG, et al. Spirit 2013 statement: defining standard protocol items for clinical trials. Ann Intern Med 2013;158:200-7.

15 The Japanese Ministry of Health Labour and Welfare. Long-Term care insurance in Japan, 2002. Available: https://www.mhlw.go.jp/english/ topics/elderly/care/index.html [Accessed 30 May 2020].

16 EuroQol Group. EuroQol--a new facility for the measurement of health-related quality of life. Health Policy 1990;16:199-208.

17 Nishimura S, Tsuchiya A, Hisashige A, et al. The development of the Japanese EuroQol instrument. Iryo To Shakai 1998;8:109-23.

18 Miyaji T, lioka Y, Kuroda Y, et al. Japanese translation and linguistic validation of the US National cancer Institute's patient-reported outcomes version of the common terminology criteria for adverse events (PRO-CTCAE). J Patient Rep Outcomes 2017;1:8.

19 Gibson MJ, Andres RO, Isaacs B, et al. The prevention of falls in later life. A report of the Kellogg international work group on the prevention of falls by the elderly. Dan Med Bull 1987;34:1-24.

20 Ravn-Nielsen LV, Duckert M-L, Lund ML, et al. Effect of an inhospital multifaceted clinical pharmacist intervention on the risk of readmission: a randomized clinical trial. JAMA Intern Med 2018;178:375-82.

21 Gnjidic D, Hilmer SN, Blyth FM, et al. Polypharmacy cutoff and outcomes: five or more medicines were used to identify communitydwelling older men at risk of different adverse outcomes. J Clin Epidemiol 2012;65:989-95.

22 Gallagher PF, O'Connor MN, O'Mahony D. Prevention of potentially inappropriate prescribing for elderly patients: a randomized controlled trial using STOPP/START criteria. Clin Pharmacol Ther 2011;89:845-54.

23 Garfinkel D, Zur-Gil S, Ben-Israel J. The war against polypharmacy: a new cost-effective geriatric-palliative approach for improving drug therapy in disabled elderly people. Isr Med Assoc J 2007;9:430-4.

24 Garfinkel D, Mangin D. Feasibility study of a systematic approach for discontinuation of multiple medications in older adults: addressing polypharmacy. Arch Intern Med 2010;170:1648-54.

25 By the 2019 American Geriatrics Society Beers Criteria $®$ Update Expert Panel. American geriatrics Society 2019 updated AGS beers Criteria for potentially inappropriate medication use in older adults. J Am Geriatr Soc 2019;67:674-94. 